\title{
CLASSIFICATION OF MONGE-AMPĖRE EQUATIONS WITH TWO VARIABLES
}

\author{
BORIS KRUGLIKOV \\ Chair of Mathematical Modeling \\ Baumann Moscow State Technological University \\ P.O.Box 546, 119618, Moscow, Russia \\ E-mail:kruglikov@glasnet.ru
}

\begin{abstract}
This paper deals with the classification of hyperbolic Monge-Ampère equations on a two-dimensional manifold. We solve the local equivalence problem with respect to the contact transformation group assuming that the equation is of general position nondegenerate type. As an application we formulate a new method of finding symmetries. This together with previous author's results allows to state the solution of the classical S. Lie equivalence problem for the Monge-Ampère equations.
\end{abstract}

1. Introduction. Monge-Ampère equations with two variables are second order nonlinear equations of the form

$$
A \frac{\partial^{2} u}{\partial x^{2}}+2 B \frac{\partial^{2} u}{\partial x \partial y}+C \frac{\partial^{2} u}{\partial y^{2}}+D\left(\frac{\partial^{2} u}{\partial x^{2}} \frac{\partial^{2} u}{\partial y^{2}}-\left(\frac{\partial^{2} u}{\partial x \partial y}\right)^{2}\right)=E,
$$

where the coefficients depend on $x, y, u=u(x, y)$ and $\frac{\partial u}{\partial x}, \frac{\partial u}{\partial y}$.

First results in investigation of the equations of this and similar for more variables type belong to Goursat and Lie ([G], [Lie]). The contemporary results and references from analytical point of view can be found in $[\mathrm{Au}]$. We will consider the geometrical approach to Monge-Ampère equations ascending to Sophus Lie. It was proposed by V. Lychagin in [Ly1]. We formulate it in Section 2. This geometric approach implies as well a pointwise classification (the classification with respect to the linear contact group acting on the tangent space to the phase space) of Monge-Ampère equations ([Ly1]). The differentiable classification (i.e. with respect to the group of differentiable contact transformations of the phase space) in the case of two variables was stated in the same year by Morimoto $([\mathrm{M}])$. In his paper complex analyticity and homogeneity conditions were assumed. In this paper we also consider classification problems but we demand no rigid conditions. We impose on the equations only a condition of general position.

1991 Mathematics Subject Classification: Primary 35A30; Secondary 53A55.

The author's research is supported by the grant INTAS 96-0713.

The paper is in final form and no version of it will be published elsewhere. 
In connection with Monge-Ampère equations, which naturally arise in geometry and physics, three types of problems may be formulated:

1. Finding solutions of Monge-Ampère equations with prescribed behavior or ramification along some fixed set, finding symmetries and conservations laws. The general methods for these problems are described in [Ly2]. We illustrate the solution technique in Section 2. We propose a new way to finding symmetries for the case of two variables.

2. Describing caustics of solutions of Monge-Ampère equations, i.e. singularities of the solutions projection to the configuration space. As shown in $[R]$ for the hyperbolic quasilinear equations their list differs from the list of usual caustics as singularities of projections for general Lagrangian (Legendrian) submanifolds. The case of Monge-Ampère equations of general type can be treated in a similar way basing on the classification results from $[\mathrm{LRC}]$.

3. Equivalence problem for the Monge-Ampère equations. We allow transformations which involve not only the coordinates but also the function and its derivatives. It means that our group upon classification is the contact transformation group of 1 -jets. Note that the equations of Monge-Ampère type form a natural class among all nonlinear second order PDEs since the local classification problem for the general case includes the following transcendental subproblem: find moduli for submanifolds in the space of quadrics with respect to the fractionally-linear transformations ([KLV]).

The equivalence problem was solved in some cases in [LRC], [Ly2]. For the mixed elliptic/hyperbolic case in the neighborhood of the point Monge-Ampère equations with two variables were classified in $[\mathrm{Ku}]$. For general equations of elliptic type the classification was achieved in [Kr1], [Kr2].

In this paper we treat hyperbolic case. The classification (Theorem 4) uses the $G$-structures theory. Actually we reduce our problem to the well-known equivalence problem for $\{e\}$-structures, i.e. frame fields on a manifold ([S]). The invariants for the last classification are structural functions, i.e. the coefficients of the commutator decompositions for the basis frame fields. Moreover our reduction of the equation to the $\{e\}$-structure is canonical in terms of the structures defining the equation and this allows to write normal forms (for example in homogeneous situation as in [Kr2] for the elliptic case).

2. Lychagin's construction. Consider the 1 -jets bundle over an $n$-dimensional orientable manifold $N: J^{1} N \rightarrow N$. The manifold $J^{1} N$ equips itself with the canonical contact structure which in local coordinate system $\left(q \in N, u \in \mathbb{R}^{1}, p \in T_{q} N\right)$ takes the form $\alpha=p d q-d u$. Every function $u \in C^{\infty}(N)$ provides us with a Legendrian section $L^{n}=j^{1} u\left(N^{n}\right)$ of this bundle: $x \mapsto j^{1} u(x)=\left(q=x, u=u(x), p=\frac{\partial u}{\partial q}(x)\right)$. Let $\theta \in \Omega^{n}\left(J^{1} N\right)$, where $\Omega^{n}=C^{\infty}\left(\Lambda^{n}\right)$ is the module of $n$-forms. Then $\Delta_{\theta}(u)=\left(j^{1} u\right)^{*} \theta \in$ $\Omega^{n}\left(N^{n}\right) \simeq C^{\infty}(N)$.

Definition 1. We call $\Delta_{\theta}(u)=0$ the Monge-Ampère equation. Its (generalized) solution is a Legendrian submanifold $L^{n} \subset J^{1} N^{n},\left.\alpha\right|_{L}=0$, such that $\left.\theta\right|_{L}=0$.

ExAmple 1. Let $n=2$. Consider $\theta=d p_{1} \wedge d q_{2}-d p_{2} \wedge d q_{1}$. Substitute $p_{i}=\frac{\partial u}{\partial q_{i}}$ to the equation $\theta=0$. We obtain the Laplace equation

$$
\Delta u=\frac{\partial^{2} u}{\partial q_{1}^{2}}+\frac{\partial^{2} u}{\partial q_{2}^{2}}=0 .
$$


Two Monge-Ampère operators $\Delta_{\theta_{1}}$ and $\Delta_{\theta_{2}}$ coincide iff $\theta_{2}=\theta_{1}+\rho_{1} \wedge \alpha+\rho_{2} \wedge d \alpha$ ([Ly2]). Actually only $\alpha$ and $d \alpha$ vanish on every Legendrian submanifold. So as the first step of the classification we need to pick up a representative in each class $\bmod (\alpha, d \alpha)$. With modulo $\alpha$ it is arranged in the following way. Assume a contact symmetry $X_{f}$ with the generating function $f \neq 0$ in the neighborhood considered, $X_{f}(\theta)=0$ ([Ly1]). Send it by a contactomorphism to the Reeb vector field: $X_{f} \mapsto X_{1}, \alpha\left(X_{1}\right)=1, d \alpha\left(X_{1}, \cdot\right)=0$. Thus $X_{1}(\theta)=0$ and we may consider $\theta$ as a form on $T^{*} N=J^{1} N / \mathbb{R}^{1}$. Still we have a class $\theta(\bmod d \alpha)$. To kill this choice take $\theta$ in the class to be an effective form, which makes the unique choice.

Definition 2. Consider a symplectic manifold $\left(M^{2 n}, \omega\right)$ and an effective $n$-form $\theta \in \Omega^{n}(M)$ on it. Let us call a pair $\left(\omega, \theta_{\text {eff }}\right)$ the generalized Monge-Ampère equation. Its solution is a Lagrangian submanifold $L^{n} \subset M^{2 n},\left.\omega\right|_{L}=0$, such that $\left.\theta\right|_{L}=0$.

For the case $n=2$ we have $\omega, \theta \in \Omega^{2}\left(M^{4}\right)$ and the effectiveness condition is equivalent to the equality $\theta \wedge \omega=0$. Two generalized Monge-Ampère equations given by forms $\theta_{1}$ and $\theta_{2}$ are equivalent if the forms are conformally symplectomorphic.

EXAmple 2. Consider the nonlinear wave equation

$$
\frac{\partial^{2} u}{\partial q_{1}^{2}}=\frac{\partial}{\partial q_{2}}\left(f\left(\frac{\partial u}{\partial q_{2}}\right)\right)
$$

It corresponds to the form $\theta=d p_{1} \wedge d q_{2}+d f\left(p_{2}\right) \wedge d q_{1}$. Making a turn by the angle $\frac{\pi}{2}$ in the plane $\left\langle p_{2}, q_{2}\right\rangle$ (which is a symplectic transformation) we obtain the form $\tilde{\theta} \stackrel{2}{=}$ $d p_{1} \wedge d p_{2}-d f\left(q_{2}\right) \wedge d q_{1}$. Now the equivalent equation $\Delta_{\tilde{\theta}}$ takes the form

$$
\operatorname{det} \operatorname{Hess} u\left(q_{1}, q_{2}\right)=-f^{\prime}\left(q_{2}\right) \text {. }
$$

And we immediately get three first series of solutions:

$$
\begin{aligned}
& \text { 1) } u=c_{0}+c_{1} q_{1}+c_{2} q_{2}+\frac{q_{1}^{2}}{2 a}-a \int f\left(q_{2}\right) d q_{2}, \\
& \text { 2) } u=q_{1} \int \sqrt{f^{\prime}\left(q_{2}\right)} d q_{2}+\Phi\left(q_{2}\right), \\
& \text { 3) } u=\frac{1}{4}\left[\frac{c_{2}+2 q_{1}}{c_{0}+c_{1} q_{2}}\right]^{2}-\frac{1}{2} \int\left[\int\left(c_{0}+c_{1} q_{2}\right) f^{\prime}\left(q_{2}\right) d q_{2}\right] d q_{2}+\left(c_{3} q_{1}+c_{4} q_{2}+c_{5}\right) .
\end{aligned}
$$

Performing now the back transformation, which is obtained by an implicit function theorem, we get series of solutions of the nonlinear wave equation.

Moreover if we make a turn in the plane $\left\langle p_{1}, q_{1}\right\rangle$, then we obtain an equivalent linear equation

$$
\frac{\partial^{2} u}{\partial q_{2}^{2}}=f^{\prime}\left(q_{2}\right) \frac{\partial^{2} u}{\partial q_{1}^{2}}
$$

3. Pointwise classification and integrability results. From now on we consider only generalized Monge-Ampère equations with two variables $(n=2)$. Let us normalize the form $\theta$ by the condition $\operatorname{Pf}(\theta)=\frac{\theta \wedge \theta}{\omega \wedge \omega}=0$ or \pm 1 . Define an automorphism $j \in T^{*} M \otimes T M$ by the formula $\theta(X, Y)=\omega(j X, Y)$. Then three cases are possible: 
- Elliptic case, $\quad \operatorname{Pf}(\theta)=1 \Leftrightarrow j^{2}=-\mathbf{1}$ (almost complex structure).

- Hyperbolic case, $\operatorname{Pf}(\theta)=-1 \Leftrightarrow j^{2}=\mathbf{1}$ (almost product structure).

- Parabolic case, $\operatorname{Pf}(\theta)=0 \Leftrightarrow j^{2}=\mathbf{0}$ (nilpotent structure).

In the almost product case the spectrum is $\operatorname{Sp}(j)=\{-1,-1,1,1\}$ and we assume that the structure is semisimple. In the nilpotent case we assume that $\operatorname{Ker}(j)=\operatorname{Im}(j)$ form a (2-dimensional) distribution.

Let us recall that the integrable geometric structure is one which is equivalent to the standard (plane) geometric structure of the considered type $([\mathrm{S}])$. Equivalently in some coordinate system the structure is given by the defining relations with constant coefficients. In particular in our case this means that the components $j_{t}^{s}$ of the operator $j$ are constant.

Let us call an operator field $j n$-integrable if it is a direct sum $j=f_{1} j_{1} \oplus \ldots \oplus f_{k} j_{k}$ under some decomposition $T M=V_{1} \oplus \ldots \oplus V_{k}$. Here $f_{\nu}$ are some functions and $j_{\nu}$ are integrable on $M$ structures acting in the integrable distributions $V_{\nu}$. Note that the notion of n-integrability is equivalent to that of integrability for almost complex and almost product structures. However it is wider in the case of nilpotent structure: the definition $j^{2}=0$ does not normalize the structure $j$.

THEOREM 1. The structure $j$ is n-integrable if and only if the Monge-Ampère equation $(\omega, \theta)$ is equivalent to one of the forms:

- Almost complex structure: $\Delta u=0$.

- Almost product structure: $\frac{\partial^{2} u}{\partial x^{2}}=\frac{\partial^{2} u}{\partial y^{2}}$.

- Nilpotent structure: $\frac{\partial^{2} u}{\partial x^{2}}=0$.

Proof. Since the necessity condition is evident we consider only the sufficiency. Consider at first the elliptic case. Since $j$ is integrable there exists a complex coordinate system $z_{1}=x_{1}+i y_{1}, z_{2}=x_{2}+i y_{2}$. Since $j \partial_{x_{k}}=\partial_{y_{k}}, k=1,2$, we have

$$
\omega\left(\partial_{x_{k}}, \partial_{y_{k}}\right)=0, \quad \omega\left(\partial_{x_{1}}, \partial_{x_{2}}\right)=-\omega\left(\partial_{y_{1}}, \partial_{y_{2}}\right)=f, \quad \omega\left(\partial_{x_{1}}, \partial_{y_{2}}\right)=\omega\left(\partial_{y_{1}}, \partial_{x_{2}}\right)=g .
$$

This means that $\omega=f\left(d x_{1} \wedge d x_{2}-d y_{1} \wedge d y_{2}\right)+g\left(d x_{1} \wedge d y_{2}+d y_{1} \wedge d x_{2}\right)$. The condition $d \omega=0$ implies that the function $h(z)=f(x, y)-i g(x, y)$ is holomorphic. In coordinates $\left(z_{1}, \bar{z}_{1}, z_{2}, \bar{z}_{2}\right)$ we have

$$
\omega=\frac{1}{2} h(z) d z_{1} \wedge d z_{2}+\frac{1}{2} \bar{h}(z) d \bar{z}_{1} \wedge d \bar{z}_{2}=\operatorname{Re}[\Omega(z)]
$$

where $\Omega(z)=h(z) d z_{1} \wedge d z_{2}$ is a complex volume form on $\mathcal{O}(0) \simeq \mathbb{R}^{4} \simeq \mathbb{C}^{2}$ in a neighborhood of $0: f(0)^{2}+g(0)^{2} \neq 0 \Rightarrow h(0) \neq 0$. By a holomorphic change of coordinates we transform our form to the $\Omega(z)=d z_{1} \wedge d z_{2}$, whence

$$
\omega=d p_{1} \wedge d q_{1}+d p_{2} \wedge d q_{2}, \quad \theta=d p_{2} \wedge d q_{1}-d p_{1} \wedge d q_{2},
$$

and we obtain the Laplace equation $\Delta u=0$.

We can similarly treat the hyperbolic case. But instead of $\mathbb{C}^{2}$ we should use $\mathbf{D}^{2}$, where $\mathbf{D}$ is the algebra of dual numbers $z=x+j y, j^{2}=\mathbf{1}(x, y \in \mathbb{R})$. We consider the dual Cauchy-Riemann equations $\frac{\partial u}{\partial x}=\frac{\partial v}{\partial y}, \frac{\partial u}{\partial y}=\frac{\partial v}{\partial x}$ and the dual volume form. Finally instead 
of the Laplace equation we get the wave equation. Another approach is to use a pair of integrable distributions - the eigenspaces of $j$.

Consider now the nilpotent case. From n-integrability condition we deduce that there exists a coordinate system $(p, q)$ such that each of 2-planes $\left\langle\partial_{p_{1}}, \partial_{p_{2}}\right\rangle,\left\langle\partial_{q_{1}}, \partial_{q_{2}}\right\rangle$ is $j$-invariant and we can multiply the structure by $f_{1}^{-1}$ along the first 2-plane and by $f_{2}^{-1}$ along the other to obtain an integrable structure. This operation does not affect the equation $\theta=0$. Let us denote this new integrable structure by the same letter $j$. Now in the coordinates: $j \partial_{p_{1}}=0, j \partial_{p_{2}}=\partial_{p_{1}}, j \partial_{q_{1}}=\partial_{q_{2}}, j \partial_{q_{2}}=0$. This implies

$$
\omega\left(\partial_{p_{1}}, \partial_{q_{2}}\right)=\omega\left(\partial_{p_{1}}, \partial_{p_{2}}\right)=\omega\left(\partial_{q_{1}}, \partial_{q_{2}}\right)=0, \quad \omega\left(\partial_{p_{k}}, \partial_{q_{k}}\right)=f, \quad \omega\left(\partial_{p_{2}}, \partial_{q_{1}}\right)=g .
$$

Thus $\omega=f\left(d p_{1} \wedge d q_{1}+d p_{2} \wedge d q_{2}\right)+g d p_{2} \wedge d q_{1}$. From the condition $d \omega=0$ we have $\frac{\partial f}{\partial p_{2}}=\frac{\partial g}{\partial p_{1}}, \frac{\partial f}{\partial q_{1}}=\frac{\partial g}{\partial q_{2}}, \frac{\partial f}{\partial p_{1}}=\frac{\partial f}{\partial q_{2}}=0$, whence

$$
g=p_{1} \frac{\partial f\left(p_{2}, q_{1}\right)}{\partial p_{2}}+q_{2} \frac{\partial f\left(p_{2}, q_{1}\right)}{\partial q_{1}}+\tilde{g}\left(p_{2}, q_{1}\right) .
$$

Therefore $\omega=d \hat{p}_{1} \wedge d \hat{q}_{1}+d \hat{p}_{2} \wedge d \hat{q}_{2}$, where $\hat{p}_{1}=p_{1} f, \hat{p}_{2}=p_{2}, \hat{q}_{1}=q_{1}, \hat{q}_{2}=q_{2} f+G$, $G\left(p_{2}, q_{1}\right)=\int \tilde{g}\left(p_{2}, q_{1}\right) d q_{1}$. In these new coordinates $j \partial_{\hat{p}_{1}}=0, j \partial_{\hat{p}_{2}}=f \partial_{\hat{p}_{1}}, j \partial_{\hat{q}_{1}}=f \partial_{\hat{q}_{2}}$, $j \partial_{\hat{q}_{2}}=0$. Thus $\theta=i_{j} \omega=f d \hat{p}_{2} \wedge d \hat{q}_{1}$, from which the claim follows (note that the conformal factor $f \neq 0$ does not affect the equation).

R e mark 1. The first two assertions of this theorem are similar to those of Theorem 1.5 from [LRC]. However the proofs are different.

4. Nijenhuis tensors and distributions. In Theorem 1 the integrability criteria can be expressed by means of the Nijenhuis tensors

$$
N_{j}(X, Y)=[j X, j Y]-j[j X, Y]-j[X, j Y]+j^{2}[X, Y] .
$$

$N_{j}$ is actually a tensor: it is straightforward to check the right hand size depends only on the values of the fields $X, Y$ at the point considered. Moreover it is the so-called Nijenhuis self-bracket of the structure $j: N_{j}=\| j, j \rrbracket$ ([FN]). Note that for every structure $j \in T^{*} M \otimes T M$ satisfying $j^{2}=$ const. 1 the following identity holds: $N_{j}(j X, Y)=$ $N_{j}(X, j Y)=-j N_{j}(X, Y)$. Now if $j$ is nondegenerate structure from Theorem 1, i.e. almost complex or almost product structure, we have the following integrability criterion:

$(\star)$ The structure $j$ is integrable if and only if $N_{j}=0$.

For almost complex structure it is Newlander-Nirenberg theorem ([NN]). For almost product it follows from Frobenius theorem and is a particular case of Haantjes theorem ([Ha]). For the nilpotent structure the condition $N_{j}=0$ does not imply integrability, but it implies n-integrability as we will see in Appendix (Section 9). So we deduce from Theorem 1

Corollary. The Monge-Ampère equation $(\omega, \theta)$ can be written in one of the forms of Theorem 1 iff for the corresponding structure $j$ we have $N_{j}=0$.

We will consider the classification of hyperbolic equations which is analogous to the classification of elliptic equations given in [Kr1]. So we are interested in almost product 
structures $j, j^{2}=\mathbf{1}$. We assume that at every point we have two independent eigenvectors of $j$ with eigenvalues +1 and two independent eigenvectors with eigenvalues -1 . Thus we define two distributions

$$
\Pi_{+}^{2}=\left\{\xi \in T_{x} M, j \xi=\xi\right\}, \quad \Pi_{-}^{2}=\left\{\xi \in T_{x} M, j \xi=-\xi\right\} .
$$

Let us denote by $P_{+}, P_{-}$the projection operators corresponding to the decomposition $T M^{4}=\Pi_{+}^{2} \oplus \Pi_{-}^{2}, P_{ \pm}^{2}=P_{ \pm}, P_{+}+P_{-}=1$. We have:

$$
\begin{aligned}
& \xi, \eta \in \Pi_{+}^{2} \Rightarrow N_{j}(\xi, \eta)=2([\xi, \eta]-j[\xi, \eta])=2(1-j)[\xi, \eta]=4 P_{-}[\xi, \eta], \\
& \xi, \eta \in \Pi_{-}^{2} \Rightarrow N_{j}(\xi, \eta)=2([\xi, \eta]+j[\xi, \eta])=2(1+j)[\xi, \eta]=4 P_{+}[\xi, \eta], \\
& \xi \in \Pi_{+}^{2}, \eta \in \Pi_{-}^{2} \Rightarrow N_{j}(\xi, \eta)=0 .
\end{aligned}
$$

Recall that fixing a distribution $\Pi$ is equivalent to fixing a differential system $\mathcal{D}(\Pi)$ of its sections, i.e. vector fields which are tangent to the distribution $\Pi$. From this submodule of the $C^{\infty}(M)$-module of vector fields we can construct the extending sequence of derived submodules: $\mathcal{D}_{1}=\mathcal{D}(\Pi), \mathcal{D}_{k+1}=\left[\mathcal{D}_{1}, \mathcal{D}_{k}\right]$. If the module $\mathcal{D}_{k}$ is projective it is a module of sections of some distribution $\Pi_{k}=\partial^{(k-1)} \Pi$. We can define the associated vector bundles $\Pi^{[k+1]}=\Pi_{k+1} / \Pi_{k}$. The Tanaka invariant $([\mathrm{T}])$ is a canonically defined graded Lie multiplication on the bundle of graded vector space $\Pi^{\star}=\bigoplus \Pi^{[k]}$ induced from the commutator $\xi, \eta \mapsto[\xi, \eta]$ by the quotient procedure.

Now if the distribution $\Pi$ is two-dimensional then the derived distribution $\partial \Pi$ is generically three-dimensional. Thus in the regular case we have two new distributions:

$$
\Pi_{+}^{3}=\partial \Pi_{+}^{2}, \quad \Pi_{-}^{3}=\partial \Pi_{-}^{2} .
$$

Therefore as a consequence of the formulas for the Nijenhuis tensor we deduce

Proposition 2. The Nijenhuis tensor of an almost product structure $j$ is completely determined by the Tanaka invariants of the distributions $\Pi_{+}^{2}$ and $\Pi_{-}^{2}: T_{ \pm}: \Pi_{ \pm}^{2} \wedge \Pi_{ \pm}^{2} \rightarrow$ $\Pi_{ \pm}^{[2]}, \operatorname{dim}\left(\Pi_{ \pm}^{[2]}\right)=1$. Thus for $\operatorname{dim} M=4$ the image of the Nijenhuis tensor is correctly defined, is two-dimensional distribution for regular distributions $\Pi_{+}^{2}, \Pi_{-}^{2}$ and satisfies the decomposition

$$
\Pi_{j}^{2}=\operatorname{Im} N_{j}=\left(\Pi_{+}^{3} \cap \Pi_{-}^{2}\right) \oplus\left(\Pi_{-}^{3} \cap \Pi_{+}^{2}\right) .
$$

Now we define the general position property for Monge-Ampère equations. We carry the classification under the fulfillment of this condition.

Definition 3. Let us call a Monge-Ampère equation $(\omega, j)$ nondegenerate in a (germ of) neighborhood $\mathcal{O}_{x}$ of the point $x \in M^{4}$ if

○ Almost product structure $j$ is nonintegrable in $\mathcal{O}_{x}$. This means that $\Pi_{j}^{2}=\operatorname{Im} N_{j}$ is a two-dimensional distribution with no singularities.

○ The distribution $\Pi_{j}^{2}$ is nonintegrable, i.e. it is not the tangent distribution to a foliation anywhere. This means that the derived distribution $\partial \Pi_{j}^{2}$ is three-dimensional.

○ The derived distribution $\partial \Pi_{j}^{2}$ at any point $x^{\prime} \in \mathcal{O}_{x}$ coincides neither with $\Pi_{+}^{3}$ nor with $\Pi_{-}^{3}$. 
5. Invariants of the pair consisting of symplectic and almost product structures. We now need to classify locally either the pair $(\omega, \theta)$ or the pair $(\omega, j)$ on a 4-dimensional manifold $M$. We can construct invariants in two ways.

First one can consider the pair $(\omega, j)$ and use local invariants of the almost product structure $j$, such as Tanaka invariants. On the other hand one can treat another representative for the generalized Monge-Ampère equation - the pair $(\omega, \theta)$ - in the following way. By the Lepage theorem ([Ly2]) the symplectic form $\omega$ divides the 3 -form $d \theta: d \theta=\omega \wedge \sigma$. By 1-form $\sigma$ one can construct forms $\theta \wedge \sigma, d \sigma$ and so on.

Let us connect these two approaches. Introduce the vector valued 2-form

$$
R_{j}^{\sigma}(X, Y)=N_{j}(X, Y)+j Y \sigma(X)-j X \sigma(Y)+Y \sigma(j X)-X \sigma(j Y) .
$$

Proposition 3. The following formula holds:

$$
R_{j}^{\sigma}=-2 j X_{\sigma} \otimes \omega,
$$

where $X_{\sigma}$ is the vector field dual to the 1-form $\sigma, \omega\left(X_{\sigma}, Z\right)=\sigma(Z)$.

Proof. Up to some signs the proof of this proposition coincides with that of Proposition 5 [Kr1], corresponding to elliptic equations. However for the completeness we present the whole argumentation. From $[\mathrm{FN}]$ we have

$$
i_{[X, Y]}=\left[L_{X}, i_{Y}\right]=\left[i_{X} d+d i_{X}, i_{Y}\right]=i_{X} d i_{Y}+d i_{X} i_{Y}-i_{Y} i_{X} d-i_{Y} d i_{X}
$$

whence

$$
\begin{aligned}
& i_{N_{j}(X, Y)} \theta= i_{[j X, j Y]} \theta-i_{j[X, j Y]} \theta-i_{j[j X, Y]} \theta+i_{[X, Y]} \theta \\
&= i_{j X} d i_{j Y} \theta+d i_{j X} i_{j Y} \theta-i_{j Y} i_{j X} d \theta-i_{j Y} d i_{j X} \theta+i_{X} d i_{Y} \theta \\
& \quad \quad d i_{X} i_{Y} \theta-i_{Y} i_{X} d \theta-i_{Y} d i_{X} \theta-i_{[X, j Y]} \omega-i_{[j X, Y]} \omega \\
&= i_{j X} d i_{Y} \omega-2 d[\theta(X, Y)]-i_{j Y} i_{j X} d \theta-i_{j Y} d i_{X} \omega+i_{X} d i_{Y} \theta \\
& \quad-i_{Y} i_{X} d \theta-i_{Y} d i_{X} \theta-i_{X} d i_{j Y} \omega-d i_{X} i_{j Y} \omega+i_{j Y} i_{X} d \omega \\
& \quad+i_{j Y} d i_{X} \omega-i_{j X} d i_{Y} \omega-d i_{j X} i_{Y} \omega+i_{Y} i_{j X} d \omega+i_{Y} d i_{j X} \omega \\
&=i_{j X} d i_{Y} \omega-2 d[\theta(X, Y)]-i_{j Y} i_{j X} d \theta-i_{j Y} d i_{X} \omega+i_{X} d i_{Y} \theta-i_{Y} i_{X} d \theta \\
& \quad-i_{Y} d i_{X} \theta-i_{X} d i_{Y} \theta+2 d[\theta(X, Y)]+i_{j Y} d i_{X} \omega-i_{j X} d i_{Y} \omega+i_{Y} d i_{X} \theta \\
&=-d \theta(X, Y, \cdot)-d \theta(j X, j Y, \cdot)=-j^{2} d \theta(X, Y, \cdot)-d \theta(j X, j Y, \cdot) .
\end{aligned}
$$

Note that this identity can also be obtained from the expression of $d \theta$ coming from the Cartan formula:

$$
\begin{aligned}
d \theta(X, Y, Z)=\partial_{X} \omega(j Y, Z)- & \partial_{j X} \omega(Y, Z) \\
& +\omega([j X, Y]-j[X, Y], Z)+\omega(Y,[j X, Z]-j[X, Z]) .
\end{aligned}
$$

Using the above expression of $i_{N_{j}(X, Y)} \theta$ and the formula $d \theta=\omega \wedge \sigma$, we have

$$
d \theta(X, Y, Z)=\omega(X, Y) \sigma(Z)+\omega(Z, X) \sigma(Y)+\omega(Y, Z) \sigma(X) .
$$

We can get a similar expression for $d \theta(j X, j Y, Z)$. Hence it follows that

$$
\begin{aligned}
\omega\left(j N_{j}(X, Y), Z\right)=\theta\left(N_{j}(X, Y), Z\right) & =-2 \omega(X, Y) \sigma(Z)-\omega(Z, X) \sigma(Y) \\
& -\omega(Y, Z) \sigma(X)-\omega(Z, j X) \sigma(j Y)-\omega(j Y, Z) \sigma(j X) .
\end{aligned}
$$


From this equality we obtain

$$
\begin{aligned}
\omega\left(j N_{j}(X, Y)+Y \sigma(X)-X \sigma(Y)+j Y \sigma(j X)-j X \sigma(j Y), Z\right) & \\
& =\omega\left(j R_{j}^{\sigma}(X, Y), Z\right)=-2 \omega(X, Y) \sigma(Z),
\end{aligned}
$$

which was to be proved.

Corollary. The almost product structure $j$ is integrable if and only if the form $\theta$ is closed: $d \theta=0$.

Proof. The proof in one direction is contained in that of Theorem 1.5 from [LRC] and follows from Proposition 3 and integrability criterion $(\star)$. Actually, if $d \theta=0$ then $\sigma=0, X_{\sigma}=0, R_{j}^{\sigma}=0$ and $N_{j}=0$. Consider now the inverse statement. Let $j$ be an integrable almost product structure, i.e. $N_{j}=0$. Suppose $\sigma \neq 0$. Then there exists a basis $(X, j X, Y, j Y)$ such that $X, j X, Y \in \operatorname{Ker} \sigma, \sigma(j Y)=1$ (in particular $X, Y \notin \Pi_{ \pm}^{2}$ ). We have $-X=R_{j}^{\sigma}(X, Y) \in\left\langle j X_{\sigma}\right\rangle$ and $-j X=R_{j}^{\sigma}(j X, Y) \in\left\langle j X_{\sigma}\right\rangle$. This contradiction shows that $\sigma=0$ and $d \theta=0$.

6. Differentiable classification: the main theorem. In this section we consider nondegenerate Monge-Ampère equations. We begin by proving that the decomposition $T M=\Pi_{+}^{2} \oplus \Pi_{-}^{2}$ is symplectic.

Lemma 1. Two subspaces $\Pi_{+}^{2}$ and $\Pi_{-}^{2}$ are symplectic and skew $\omega$-orthogonal.

Proof. Actually for every $X \in \Pi_{+}^{2}$ and $Y \in \Pi_{-}^{2}$ we have

$$
\omega(X, Y)=\omega(j X, Y)=\omega(X, j Y)=-\omega(X, Y)=0 .
$$

Thus the claim follows from the nondegeneracy of $\omega$.

Let us define 1-dimensional distributions $\Pi_{ \pm}^{1}$ by the formulas

$$
\Pi_{+}^{1}=\Pi_{+}^{2} \cap \Pi_{-}^{3}, \quad \Pi_{-}^{1}=\Pi_{-}^{2} \cap \Pi_{+}^{3} .
$$

Define also another 1-distribution $\Gamma_{\sigma}^{1}=\left\langle X_{\sigma}\right\rangle=\left\{\lambda X_{\sigma} \mid \lambda \in \mathbb{R}\right\}$. We have the following inclusions:

Lemma 2. $\Gamma_{\sigma}^{1} \subset \Pi_{j}^{2} \subset \operatorname{Ker} \sigma=\left(\Gamma_{\sigma}^{1}\right)^{\perp \omega}$.

Proof. First note that since $\sigma\left(X_{\sigma}\right)=0$ we have $\sigma\left(N_{j}(X, Y)\right)=\sigma\left(j N_{j}(j Y, X)\right)=$ $\sigma\left(j R_{j}^{\sigma}(j Y, X)\right)=0$. Thus $\left.\sigma\right|_{\operatorname{Im} N_{j}}=0$ and $\Pi_{j}^{2} \subset \operatorname{Ker} \sigma$. Next note that $\Pi_{j}^{2}$ is a Lagrangian plane because $\Pi_{j}^{2}=\Pi_{+}^{1} \oplus \Pi_{-}^{1}$ and the 1 -distributions $\Pi_{+}^{1}$ and $\Pi_{-}^{1}$ are $\omega$-orthogonal. From the definition of $X_{\sigma}$ we deduce $\Gamma_{\sigma}^{1}=\operatorname{Ker}\left(\left.\omega\right|_{\operatorname{Ker} \sigma}\right)$. Now the claim follows from the fact that in a three-dimensional space equipped with a nonzero 2-form $\omega$ the kernel of $\omega$ lies in every isotropic 2 -space.

LEMma 3. $\Pi_{+}^{1}=\operatorname{Ker}\left(\left.\omega\right|_{\Pi_{-}^{3}}\right)$ and $\Pi_{-}^{1}=\operatorname{Ker}\left(\left.\omega\right|_{\Pi_{+}^{3}}\right)$.

Proof. Let $X \in \Pi_{+}^{1} \backslash\{0\}$. Since by Lemma 1 the 1 -form $\omega(X, \cdot)$ vanishes on $\Pi_{-}^{2}$ and since it trivially vanishes on $\Pi_{+}^{1}$ the vector $X$ is skew $\omega$-orthogonal to the whole $\Pi_{-}^{3}$ and thus lies in the kernel of the restriction of $\omega$. For $\Pi_{-}^{1}$ the arguments are similar. 
Note that by definition of the plane $\Pi_{j}^{2}=\operatorname{Im} N_{j}$ for every nonzero vectors $X_{+} \in \Pi_{+}^{1}$, $Y_{-} \in \Pi_{-}^{1}$ there exist vectors $Z_{1}, Z_{2}$ such that

$$
N_{j}\left(X_{+}, Z_{1}\right)=Y_{-}, \quad N_{j}\left(Y_{-}, Z_{2}\right)=X_{+} .
$$

Moreover since $\Pi_{+}^{2}$ and $\Pi_{-}^{2}$ are $N_{j}$-orthogonal we can choose $Z_{1} \in \Pi_{+}^{2} \backslash \Pi_{+}^{1}$ and $Z_{2} \in \Pi_{-}^{2} \backslash \Pi_{-}^{1}$. Also imposing the conditions

$$
\omega\left(X_{+}, Z_{1}\right)=1, \quad \omega\left(Y_{-}, Z_{2}\right)=1
$$

we uniquely determine $X_{+}, Y_{-}$and we determine $Z_{1}\left(\bmod \Pi_{+}^{1}\right), Z_{2}\left(\bmod \Pi_{-}^{1}\right)$. Now since we assumed our Monge-Ampère equation nondegenerate we have for the vector fields $X_{+}$ and $Y_{-}$defined in $\mathcal{O}_{x}:\left[X_{+}, Y_{-}\right] \notin \Pi_{j}^{2}$. Moreover due to the third condition of nondegeneracy of the Monge-Ampère equation $\left[X_{+}, Y_{-}\right] \notin \Pi_{ \pm}^{3}$. Thus $P_{ \pm}\left[X_{+}, Y_{-}\right] \notin \Pi_{ \pm}^{1}$ and we can uniquely determine the vectors $Z_{1}, Z_{2}$ by the conditions

$$
\begin{array}{ll}
Z_{+} \equiv Z_{1}\left(\bmod \Pi_{+}^{1}\right), & Z_{+} \in \mathbb{R} \cdot P_{+}\left(\left[X_{+}, Y_{-}\right]\right), \\
Z_{-} \equiv Z_{2}\left(\bmod \Pi_{-}^{1}\right), & Z_{-} \in \mathbb{R} \cdot P_{-}\left(\left[X_{+}, Y_{-}\right]\right) .
\end{array}
$$

Lemma 4. $\sigma\left(Z_{+}\right)=-\frac{1}{2}, \sigma\left(Z_{-}\right)=\frac{1}{2}$.

Pro of. From Proposition 3 and the properties of $\left(X_{+}, Y_{-}, Z_{+}, Z_{-}\right)$we have

$$
\begin{aligned}
R_{j}^{\sigma}\left(X_{+}, Z_{+}\right) & =Y_{-}+j Z_{+} \sigma\left(X_{+}\right)-j X_{+} \sigma\left(Z_{+}\right)+Z_{+} \sigma\left(j X_{+}\right)-X_{+} \sigma\left(j Z_{+}\right) \\
& =Y_{-}-2 \sigma\left(Z_{+}\right) X_{+}=-2 j X_{\sigma}, \\
R_{j}^{\sigma}\left(Y_{-}, Z_{-}\right) & =X_{+}+j Z_{-} \sigma\left(Y_{-}\right)-j Y_{-} \sigma\left(Z_{-}\right)+Z_{-} \sigma\left(j Y_{-}\right)-Y_{-} \sigma\left(j Z_{-}\right) \\
& =X_{+}+2 \sigma\left(Z_{-}\right) Y_{-}=-2 j X_{\sigma} .
\end{aligned}
$$

Thus

$$
X_{\sigma}=\sigma\left(Z_{+}\right) X_{+}+\frac{1}{2} Y_{-}=-\frac{1}{2} X_{+}+\sigma\left(Z_{-}\right) Y_{-} .
$$

Since $X_{+}$and $Y_{-}$are linearly independent we have $X_{\sigma}=-\frac{1}{2} X_{+}+\frac{1}{2} Y_{-}, \sigma\left(Z_{+}\right)=-\frac{1}{2}$, $\sigma\left(Z_{-}\right)=\frac{1}{2}$.

Let us define the $\{e\}$-structure, i.e. basis frame on $\mathcal{O}_{x}$, by the formulas

$$
P_{1}=X_{+}, \quad P_{2}=Y_{-}, \quad Q_{1}=Z_{+}, \quad Q_{2}=Z_{-} .
$$

Theorem 4. A nondegenerate generalized hyperbolic Monge-Ampère equation $(\omega, j)$ canonically determines an $\{e\}$-structure, i.e. the field of basis frames $(P, Q)$. This structure is a complete invariant, i.e. two nondegenerate hyperbolic Monge-Ampère equations are isomorphic iff the corresponding $\{e\}$-structures are. The classifying $\{e\}$-structure satisfies the following relations:

\begin{tabular}{|c||c|c|c|c|}
\hline$\omega(\uparrow, \leftarrow)$ & $P_{1}$ & $P_{2}$ & $Q_{1}$ & $Q_{2}$ \\
\hline \hline$P_{1}$ & 0 & 0 & 1 & 0 \\
\hline$P_{2}$ & 0 & 0 & 0 & 1 \\
\hline$Q_{1}$ & -1 & 0 & 0 & 0 \\
\hline$Q_{2}$ & 0 & -1 & 0 & 0 \\
\hline
\end{tabular}

\begin{tabular}{|c||c|c|c|c|}
\hline$N_{j}(\uparrow, \leftarrow)$ & $P_{1}$ & $P_{2}$ & $Q_{1}$ & $Q_{2}$ \\
\hline \hline$P_{1}$ & 0 & 0 & $P_{2}$ & 0 \\
\hline$P_{2}$ & 0 & 0 & 0 & $P_{1}$ \\
\hline$Q_{1}$ & $-P_{2}$ & 0 & 0 & 0 \\
\hline$Q_{2}$ & 0 & $-P_{1}$ & 0 & 0 \\
\hline
\end{tabular}




\begin{tabular}{|c||c|c|c|c|}
\hline$\theta(\uparrow, \leftarrow)$ & $P_{1}$ & $P_{2}$ & $Q_{1}$ & $Q_{2}$ \\
\hline \hline$P_{1}$ & 0 & 0 & 1 & 0 \\
\hline$P_{2}$ & 0 & 0 & 0 & -1 \\
\hline$Q_{1}$ & -1 & 0 & 0 & 0 \\
\hline$Q_{2}$ & 0 & 1 & 0 & 0 \\
\hline
\end{tabular}

\begin{tabular}{|c||c|c|c|c|}
\hline$X$ & $P_{1}$ & $P_{2}$ & $Q_{1}$ & $Q_{2}$ \\
\hline \hline$j X$ & $P_{1}$ & $-P_{2}$ & $Q_{1}$ & $-Q_{2}$ \\
\hline
\end{tabular}

\begin{tabular}{|c|c|c|c|c|}
\hline$X$ & $P_{1}$ & $P_{2}$ & $Q_{1}$ & $Q_{2}$ \\
\hline \hline$\sigma(X)$ & 0 & 0 & $-1 / 2$ & $1 / 2$ \\
\hline
\end{tabular}

Proof. The tables for $\omega, j, N_{j}$ and $\sigma$ follow from the construction of the $\{e\}$-structure and Lemmata $1-4$. They imply the table for $\theta$. Conversely given an $\{e\}$-structure satisfying the tables of the theorem we define the Monge-Ampère equation by $\omega$ and $\theta$. However note that this $\{e\}$-structure is not arbitrary, it must satisfy two conditions. First, the 2 -form $\omega$ defined by the table must be closed. Second, the Nijenhuis tensor given by the table must coincide with the tensor calculated from the almost complex structure $j$ given by the table. Equivalently we can replace one of these conditions by the coincidence condition of the 3 -form $\omega \wedge \sigma$ determined by the tables and 3 -form $d \theta$ with $\theta$ determined by the $\{e\}$-structure and the table. These two conditions stand for holonomy conditions and under their fulfillments given $\{e\}$-structure $\left(P_{1}, P_{2}, Q_{1}, Q_{2}\right)$ uniquely determines the Monge-Ampère equation.

Recall that by standard procedure the complete set of invariants for an $\{e\}$-structure $\left\{e_{i}\right\}_{i=1}^{4}$ is given by the set of structure coefficients $c_{j k}^{i}$ from the decomposition $\left[e_{j}, e_{k}\right]=$ $\sum_{i} c_{j k}^{i} e_{i}$.

The classification theorem gives also a method to computing symmetries - vector fields, the phase flow of which moves the equation to itself:

Corollary. Every symmetry of a nondegenerate Monge-Ampère equation $(\omega, j)$ is the symmetry of its canonical $\{e\}$-structure $\left(P_{1}, P_{2}, Q_{1}, Q_{2}\right)$ and vice versa. This solves the problem of finding the Lie algebra of symmetries of a nondegenerate hyperbolic (or elliptic) Monge-Ampère equation.

Actually the symmetry problem is reduced to finding symmetries of the structure functions to the canonical $\{e\}$-structure. Since such symmetry vector fields must be tangent to the level lines of the functions the problem of finding symmetries (if there are any) becomes trivial.

7. Examples and extension of the classification. We have considered nondegenerate equations, but many interesting cases become "degenerate" in the sense of Definition 3. The simplest example of such an equation is a violation of the first condition from this definition. If $\operatorname{Im} N_{j}=0$, i.e. the structure $j$ is integrable, then due to Theorem 1 the Monge-Ampère equation can be transformed to the unique normal form. Consider now some examples when the first condition holds but two others are not necessarily satisfied.

Example 3. Consider the equation

$$
\sin \varphi \cdot\left(\frac{\partial^{2} u}{\partial x^{2}}-\frac{\partial^{2} u}{\partial y^{2}}\right)=\cos \varphi \cdot \frac{\partial^{2} u}{\partial x \partial y},
$$


where $\varphi$ is a function of $x, y, \partial u / \partial x, \partial u / \partial y$. The equation corresponds to the pair $\omega=d p_{1} \wedge d q_{1}+d p_{2} \wedge d q_{2}, \theta=\cos \varphi \cdot\left(d p_{1} \wedge d q_{1}-d p_{2} \wedge d q_{2}\right)+\sin \varphi \cdot\left(d p_{1} \wedge d q_{2}+d p_{2} \wedge d q_{1}\right)$. This pair satisfies $\operatorname{Pf}(\theta)=-1, \theta \wedge \omega=0$. Moreover the 1-form $\sigma=d \theta / \omega \neq 0$. Thus the corresponding almost product structure is nonintegrable. It has the following form:

$$
\begin{array}{ll}
j \partial_{p_{1}}=\cos \varphi \cdot \partial_{p_{1}}+\sin \varphi \cdot \partial_{p_{2}}, & j \partial_{q_{1}}=\cos \varphi \cdot \partial_{q_{1}}+\sin \varphi \cdot \partial_{q_{2}}, \\
j \partial_{p_{2}}=\sin \varphi \cdot \partial_{p_{1}}-\cos \varphi \cdot \partial_{p_{2}}, & j \partial_{q_{2}}=\sin \varphi \cdot \partial_{q_{1}}-\cos \varphi \cdot \partial_{q_{2}} .
\end{array}
$$

Distributions of 2-planes which correspond to the $j$-invariant decomposition of $T^{4}$ have the form $\Pi_{+}^{2}=\left\langle a_{+}^{p}, a_{+}^{q}\right\rangle, \Pi_{-}^{2}=\left\langle a_{-}^{p}, a_{-}^{q}\right\rangle$, where

$$
\begin{array}{ll}
a_{+}^{p}=\cos \frac{\varphi}{2} \cdot \partial_{p_{1}}+\sin \frac{\varphi}{2} \cdot \partial_{p_{2}}, & a_{+}^{q}=\cos \frac{\varphi}{2} \cdot \partial_{q_{1}}+\sin \frac{\varphi}{2} \cdot \partial_{q_{2}}, \\
a_{-}^{p}=\sin \frac{\varphi}{2} \cdot \partial_{p_{1}}-\cos \frac{\varphi}{2} \cdot \partial_{p_{2}}, & a_{-}^{q}=\sin \frac{\varphi}{2} \cdot \partial_{q_{1}}-\cos \frac{\varphi}{2} \cdot \partial_{q_{2}} .
\end{array}
$$

Now $\Pi_{+}^{3}=\left\langle a_{+}^{p}, a_{+}^{q}, a_{+}^{p q}\right\rangle, \Pi_{-}^{3}=\left\langle a_{-}^{p}, a_{-}^{q}, a_{-}^{p q}\right\rangle$, with

$$
\begin{aligned}
& a_{+}^{p q}=\left[a_{+}^{p}, a_{+}^{q}\right]=-\frac{1}{2}\left(\cos \frac{\varphi}{2} \cdot \frac{\partial \varphi}{\partial p_{1}}+\sin \frac{\varphi}{2} \cdot \frac{\partial \varphi}{\partial p_{2}}\right) a_{-}^{q}+\frac{1}{2}\left(\cos \frac{\varphi}{2} \cdot \frac{\partial \varphi}{\partial q_{1}}+\sin \frac{\varphi}{2} \cdot \frac{\partial \varphi}{\partial q_{2}}\right) a_{-}^{p}, \\
& a_{-}^{p q}=\left[a_{-}^{p}, a_{-}^{q}\right]=\frac{1}{2}\left(\sin \frac{\varphi}{2} \cdot \frac{\partial \varphi}{\partial p_{1}}-\cos \frac{\varphi}{2} \cdot \frac{\partial \varphi}{\partial p_{2}}\right) a_{+}^{q}-\frac{1}{2}\left(\sin \frac{\varphi}{2} \cdot \frac{\partial \varphi}{\partial q_{1}}-\cos \frac{\varphi}{2} \cdot \frac{\partial \varphi}{\partial q_{2}}\right) a_{+}^{p} .
\end{aligned}
$$

Thus $\Pi_{+}^{1}=\Pi_{+}^{2} \cap \Pi_{-}^{3}=\left\langle a_{-}^{p q}\right\rangle, \Pi_{-}^{1}=\Pi_{-}^{2} \cap \Pi_{+}^{3}=\left\langle a_{+}^{p q}\right\rangle$ and $\Pi_{j}^{2}=\left\langle a_{+}^{p q}, a_{-}^{p q}\right\rangle$.

Note that for some choices of the function $\varphi$, for example for the linear case $\varphi=\varphi(q)$, the 2 -distribution $\Pi_{j}^{2}$ is integrable and our Monge-Ampère equation becomes degenerate in the sense of Definition 3, but in general it is nondegenerate and we have all invariants from Theorem 4.

ExAmPLE 4. Let $\omega=d p_{1} \wedge d q_{1}+d p_{2} \wedge d q_{2}, \theta=d p_{1} \wedge d q_{1}-d p_{2} \wedge d q_{2}+S d p_{1} \wedge d q_{2}+$ $T d p_{1} \wedge d p_{2}$. This case is also hyperbolic: $\operatorname{Pf} \theta=-1, \theta \wedge \omega=0, \sigma=d \theta / \omega \neq 0$. The computation with the Nijenhuis tensor gives

$$
\begin{aligned}
& \Pi_{+}^{2}=\left\langle\partial_{q_{1}}, \partial_{p_{1}}+\frac{S}{2} \partial_{p_{2}}-\frac{T}{2} \partial_{q_{2}}\right\rangle, \quad \Pi_{-}^{2}=\left\langle\partial_{q_{2}}-\frac{S}{2} \partial_{q_{1}}, \partial_{p_{2}}-\frac{T}{2} \partial_{q_{1}}\right\rangle \\
& \Pi_{+}^{3}=\left\langle\partial_{q_{1}}, \partial_{p_{1}}+\frac{S}{2} \partial_{p_{2}}-\frac{T}{2} \partial_{q_{2}}, \frac{\partial S}{\partial q_{1}} \partial_{p_{2}}-\frac{\partial T}{\partial q_{1}} \partial_{q_{2}}\right\rangle, \quad \Pi_{-}^{3}=\left\langle\partial_{q_{1}}, \partial_{q_{2}}, \partial_{p_{2}}\right\rangle .
\end{aligned}
$$

Thus

$$
\begin{gathered}
\Pi_{+}^{1}=\left\langle\partial_{q_{1}}\right\rangle, \quad \Pi_{-}^{1}=\left\langle\left(\frac{S}{2} \frac{\partial T}{\partial q_{1}}-\frac{T}{2} \frac{\partial S}{\partial q_{1}}\right) \partial_{q_{1}}+\frac{\partial S}{\partial q_{1}} \partial_{p_{2}}-\frac{\partial T}{\partial q_{1}} \partial_{q_{2}}\right\rangle ; \\
\Pi_{j}^{2}=\left\langle\partial_{q_{1}}, \frac{\partial S}{\partial q_{1}} \partial_{p_{2}}-\frac{\partial T}{\partial q_{1}} \partial_{q_{2}}\right\rangle .
\end{gathered}
$$

It's easy to see that in this case $\partial \Pi_{j}^{2}=\Pi_{-}^{3}$ and thus our case is nonintegrable but is degenerate in sense of Definition 3.

Now we extend our classification to the case when $\Pi_{j}^{2}$ is still nonintegrable distribution and its first derivative distribution is either $\Pi_{+}^{3}$ or $\Pi_{-}^{3}$. The construction of $X_{+}, Y_{-}$is invariable, we have only to pick $Z_{+}, Z_{-}$. Let for example $\partial \Pi_{j}^{2}=\Pi_{+}^{3}$. Then $\left[X_{+}, Y_{-}\right] \in \Pi_{+}^{3}$. Let us fix the unique representative $Z_{+}$for $Z_{1}$ in this direction. Now consider the 3-plane $\Pi_{-}^{3}=\left\langle X_{+}, Y_{-}, Z_{2}\right\rangle$. It is nonintegrable due to the commutation relation $\left[X_{+}, Y_{-}\right] \in\left\langle Z_{+}\right\rangle$. 
Thus the Tanaka mapping (graduate Lie multiplication) $T: \wedge^{2} \Pi_{-}^{3} \rightarrow T M^{4} / \Pi_{-}^{3}$ has a onedimensional kernel $\operatorname{Ker}(T) \subset \Pi_{-}^{3}$. Since $\left[X_{+}, Y_{-}\right] \notin \Pi_{-}^{3}$ we have $\operatorname{Ker}(T) \not \subset \Pi_{j}^{2}=\left\langle X_{+}, Y_{-}\right\rangle$. Thus $P_{-}(\operatorname{Ker}(T)) \subset \Pi_{-}^{2} \backslash \Pi_{-}^{1}$ and we choose the unique representative $Z_{-}$for $Z_{2}$ in this direction. Now by the same arguments as in Section 6 we get

Proposition 5. The statement of Theorem 4 holds for the general case when the distribution $\Pi_{j}^{2}$ is nonintegrable.

EXAMPle $4^{\prime}$ (continuation of 4 ). Now some more computations give (we omit the long expression for $\left.Z_{+}\right)$:

where

$$
\begin{gathered}
X_{+}=\Phi \cdot \partial_{q_{1}}, \quad Y_{-}=2 \frac{\partial T}{\partial q_{1}} \partial_{q_{2}}-2 \frac{\partial S}{\partial q_{1}} \partial_{p_{2}}+\left(T \frac{\partial S}{\partial q_{1}}-S \frac{\partial T}{\partial q_{1}}\right) \partial_{q_{1}} \\
Z_{-}=\frac{1}{4 \Phi \Psi}\left[2 \Phi\left(\frac{\partial^{2} T}{\partial q_{1}^{2}} \partial_{q_{2}}-\frac{\partial^{2} S}{\partial q_{1}^{2}} \partial_{p_{2}}\right)-\left(\Phi\left(S \frac{\partial^{2} T}{\partial q_{1}^{2}}-T \frac{\partial^{2} S}{\partial q_{1}^{2}}\right)+\left(Y_{-} \cdot \Phi\right)\right) \partial_{q_{1}}\right]
\end{gathered}
$$

$$
\Phi=2 \frac{\partial T}{\partial q_{2}}-2 \frac{\partial S}{\partial p_{2}}+T \frac{\partial S}{\partial q_{1}}-S \frac{\partial T}{\partial q_{1}}, \quad \Psi=\frac{\partial^{2} S}{\partial q_{1}^{2}} \frac{\partial T}{\partial q_{1}}-\frac{\partial^{2} T}{\partial q_{1}^{2}} \frac{\partial S}{\partial q_{1}} .
$$

\section{Semi-integrable equations and other degenerate cases}

Definition 4. We call a Monge-Ampère equation $(\omega, j)$ semi-integrable if for this pair all distributions $\Pi_{j}^{2}, \Pi_{+}^{3}$ and $\Pi_{-}^{3}$ are integrable.

THEOREM 6. If a generalized hyperbolic Monge-Ampère equation is semi-integrable then it has the form

$$
\frac{\partial^{2} u}{\partial x \partial y}=\frac{1}{2} A\left(u, \frac{\partial u}{\partial x}, \frac{\partial u}{\partial y}\right) \cdot \frac{\partial^{2} u}{\partial x^{2}} .
$$

Proof. Note that if every distribution indicated in the definition is integrable then they all can be rectified simultaneously (Section 9). Thus we may assume that in some local coordinates $\left(p_{1}, p_{2}, q_{1}, q_{2}\right)$ we have

$$
\Pi_{j}^{2}=\left\langle\partial_{q_{1}}, \partial_{p_{2}}\right\rangle, \quad \Pi_{+}^{3}=\left\langle\partial_{q_{1}}, \partial_{p_{2}}, \partial_{p_{1}}\right\rangle, \quad \Pi_{-}^{3}=\left\langle\partial_{q_{1}}, \partial_{p_{2}}, \partial_{q_{2}}\right\rangle .
$$

Note also that together with rectifying the three distributions we straighten the canonical 1-dimensional distributions: $\Pi_{+}^{1}=\left\langle\partial_{q_{1}}\right\rangle, \Pi_{-}^{1}=\left\langle\partial_{p_{2}}\right\rangle$. Therefore the invariant 2-distributions have the form

$$
\Pi_{+}^{2}=\left\langle\partial_{q_{1}}, \partial_{p_{1}}+\frac{A}{2} \partial_{p_{2}}\right\rangle, \quad \Pi_{-}^{2}=\left\langle\partial_{p_{2}}, \partial_{q_{2}}+\frac{B}{2} \partial_{q_{1}}\right\rangle .
$$

Thus we know the almost product structure

$$
\begin{array}{ll}
j \partial_{q_{1}}=\partial_{q_{1}}, & j \partial_{p_{1}}=\partial_{p_{1}}+A \partial_{p_{2}}, \\
j \partial_{p_{2}}=-\partial_{p_{2}}, & j \partial_{q_{2}}=-\partial_{q_{2}}-B \partial_{q_{1}} .
\end{array}
$$

Now due to Lemma 3 we have $\left.\omega\right|_{\Pi_{+}^{3}}=R_{1} d p_{1} \wedge d q_{1},\left.\omega\right|_{\Pi_{-}^{3}}=R_{2} d p_{2} \wedge d q_{2}$. Hence

$$
\omega=R_{1} d p_{1} \wedge d q_{1}+R_{2} d p_{2} \wedge d q_{2}+S d p_{1} \wedge d q_{2} .
$$

From the condition $d \omega=0$ we deduce that

$$
\frac{\partial R_{1}}{\partial q_{2}}=\frac{\partial S}{\partial q_{1}}, \quad \frac{\partial R_{2}}{\partial p_{1}}=\frac{\partial S}{\partial p_{2}}, \quad \frac{\partial R_{1}}{\partial p_{2}}=0, \quad \frac{\partial R_{2}}{\partial q_{1}}=0 .
$$


This implies $\frac{\partial^{2} S}{\partial q_{1} \partial p_{2}}=0$, whence $S=S_{1}+S_{2}, S_{1}=S_{1}\left(q_{1}, p_{1}, q_{2}\right), S_{2}=S_{2}\left(p_{2}, p_{1}, q_{2}\right)$. Thus

$$
\omega=d p_{1} \wedge\left(R_{1} d q_{1}+S_{1} d q_{2}\right)+\left(R_{2} d p_{2}+S_{2} d p_{1}\right) \wedge d q_{2} .
$$

Now since the form $\omega$ is closed, by two consecutive changes of coordinates

$$
q_{1} \mapsto Q_{1}\left(q_{1}, p_{1}, q_{2}\right), \quad p_{2} \mapsto P_{2}\left(p_{2}, p_{1}, q_{2}\right)
$$

we obtain the Darboux normal form of the symplectic structure in new coordinates, which we still denote by $(p, q): \omega=d p_{1} \wedge d q_{1}+d p_{2} \wedge d q_{2}$. This change of coordinates induces the change of coordinate basis frame:

$$
\partial_{q_{1}} \mapsto \sigma_{1} \partial_{q_{1}}, \quad \partial_{p_{1}} \mapsto \partial_{p_{1}}+\alpha \partial_{p_{2}}+\beta \partial_{q_{1}}, \quad \partial_{q_{2}} \mapsto \partial_{q_{2}}+\gamma \partial_{p_{2}}+\delta \partial_{q_{1}}, \quad \partial_{p_{2}} \mapsto \sigma_{2} \partial_{p_{2}} .
$$

Henceforth the formula for the complex multiplication in the new basis frame is similar to that of the old basis frame. Now let us use the condition $\omega\left(j \partial_{p_{1}}, \partial_{q_{2}}\right)=\omega\left(\partial_{p_{1}}, j \partial_{q_{2}}\right)$. It implies easily that $A+B=0$, whence

$$
\theta=i_{j} \omega=d p_{1} \wedge d q_{1}-d p_{2} \wedge d q_{2}+A d p_{1} \wedge d q_{2},
$$

which proves the theorem.

Now consider some other cases which also do not satisfy Definition 3. In this article we consider only regular points of Monge-Ampère equations, i.e. such that all differential systems $\Pi$ evolved are of locally constant rank; in other words they define distributions. Our consideration of Monge-Ampère equations relies on the behavior of the distribution $\Pi_{j}$. If we have no such distribution (it is 0-dimensional), we are in integrable situation considered in Theorem 1 . Note that the next and only possible case is $\operatorname{dim}\left(\Pi_{j}\right)_{x}=2$. We explain this in details in the next section. Thus there are only two cases to be considered:

1. $\Pi_{j}^{2}$ is integrable but $\Pi_{+}^{3}, \Pi_{-}^{3}$ are not.

2. $\Pi_{j}^{2}$ is integrable as well as exactly one of the distributions $\Pi_{+}^{3}, \Pi_{-}^{3}$.

Now using the argumentation similar to that of Sections 6-7 one can obtain partial invariants similar to ones from Theorem 4, which allow to find symmetries. Also note that Weinstein-Givental theorem ([AG]) implies that symplectic form is standard in the neighborhood of leaves of Lagrangian foliation defined by the integrable distribution $\Pi_{j}^{2}$.

Re mark 2. Consider 3-distribution Ker $\sigma$. For nondegenerate Monge-Ampère equations this distribution coincides neither with $\Pi_{+}^{3}$ nor with $\Pi_{-}^{3}$. This follows from the formula $X_{\sigma}=-\frac{1}{2} X_{+}+\frac{1}{2} Y_{-}$obtained in the proof of Lemma 4 and Lemmata 2 and 3. In degenerate cases $\sigma$ can be zero on $\Pi_{+}^{3}$ or $\Pi_{-}^{3}$. It is also interesting to consider the case of integrable distribution $\operatorname{Ker} \sigma$. This case is equivalently defined by the condition $d \sigma=0$ and in this situation we talk of divergence-free Monge-Ampère equations [Ly2].

9. Appendix: some relevant integrability results. Here we prove some lemmata which were used in the article.

First let us prove that if an almost product structure $j$ is nonintegrable then the distribution $\Pi_{j}$ is 2-dimensional. A priori we can assume the situation when $\Pi_{+}^{2}$ is integrable and $\Pi_{-}^{2}$ is not (or otherwise), implying $\Pi_{j}=\Pi_{+}^{1}$ being 1-dimensional, but it is impossible. 
LEMMA 5. The integrability of the distribution $\Pi_{+}^{2}$ is equivalent to the integrability of the distribution $\Pi_{-}^{2}$.

Proof. Assuming integrability $\Pi_{+}^{2}$ by the Weinstein-Givental theorem we deduce there exist local coordinates $(p, q)$ such that $\omega=d p_{1} \wedge d q_{1}+d p_{2} \wedge d q_{2}$ and $\Pi_{+}^{2}=T_{*}\left\{p_{2}=\right.$ const., $q_{2}=$ const. $\}$. Since $\Pi_{-}^{2}=\left(\Pi_{+}^{2}\right)^{\omega \perp}$ it has the form $T_{*}\left\{p_{1}=\right.$ const., $q_{1}=$ const. $\}$ and thus is also integrable.

Next we prove the statement used in Section 8.

LEMMA 6. If two transversal distributions $\Pi_{+}^{3}$ and $\Pi_{-}^{3}$ in a 4-dimensional manifold are integrable then their intersection is also integrable and they all can be rectified simultaneously.

Proof. This fact is almost evident: the distributions are tangent planes to some local foliations $\mathcal{F}_{1}$ and $\mathcal{F}_{2}$ which are transversal and hence define the 2-dimensional foliation-intersection.

Now consider the case of nilpotent structure $j, j^{2}=0$. We prove that such a structure with $N_{j}=0$ is n-integrable.

LEMmA 7. If the Nijenhuis tensor of nilpotent structure $j$ with $\operatorname{dim}[\operatorname{Ker}(j)=\operatorname{Im}(j)]=2$ vanishes, $N_{j}=0$, then the structure has the following form in some coordinate frame:

$$
j \partial_{1}=0, \quad j \partial_{2}=0, \quad j \partial_{3}=a\left(x_{1}, x_{3}, x_{4}\right) \partial_{1}, \quad j \partial_{4}=b\left(x_{2}, x_{3}, x_{4}\right) \partial_{2} .
$$

Proof. First let us deduce from the condition $N_{j}=0$ that the distribution $\operatorname{Ker}(j)=$ $\operatorname{Im}(j)$ is integrable. Actually let $\xi \in \operatorname{Ker}(j), \xi \neq 0$ and $\eta \notin j^{-1}(\xi)$. Then $\xi, j \eta$ form a basis of $\operatorname{Im}(j)$. We have

$$
0=N_{j}(\xi, \eta)=[j \xi, j \eta]-j[j \xi, \eta]-j[\xi, j \eta]=-j[\xi, j \eta]
$$

or equivalently $[\xi, j \eta] \in \operatorname{Ker}(j)$. Thus from Frobenius theorem the integrability follows.

Let this distribution integrate to the foliation $\left\{x_{3}=\right.$ const $_{1}, x_{4}=$ const $\left._{2}\right\}$. Thus our structure is given in the neighborhood of $0 \in \mathbb{R}^{4}$ by the formulas

$$
j \partial_{1}=0, \quad j \partial_{2}=0, \quad j\left(\alpha \partial_{3}+\beta \partial_{4}\right)=\partial_{1}, \quad j\left(\gamma \partial_{3}+\delta \partial_{4}\right)=\partial_{2},
$$

where the matrix of functions

$$
A=\left(\begin{array}{ll}
\alpha & \beta \\
\gamma & \delta
\end{array}\right)
$$

is nondegenerate in some neighborhood of zero, $\operatorname{det}(A) \neq 0$. These relations determine $j$ uniquely. Let us find two 1-distributions on the plane $\left\langle\partial_{1}, \partial_{2}\right\rangle$ such that their preimages by $j$ be integrable 3 -distributions. If 1-distribution is given by $\left\langle f \partial_{1}+g \partial_{2}\right\rangle$ then

$$
j^{-1}\left(\left\langle f \partial_{1}+g \partial_{2}\right\rangle\right)=\left\langle\partial_{1}, \partial_{2},(\alpha f+\gamma g) \partial_{3}+(\beta f+\delta g) \partial_{4}\right\rangle .
$$

This distribution is integrable in the following two cases: $(f, g)=(\gamma,-\alpha)$ or $(f, g)=$ $(\delta,-\beta)$. Consider two foliations of the plane $\left\langle\partial_{1}, \partial_{2}\right\rangle$ by the integral curves of the above two distributions

$$
\Sigma_{(1)}^{1}=\left\langle\gamma \partial_{1}-\alpha \partial_{2}\right\rangle, \quad \Sigma_{(2)}^{1}=\left\langle\delta \partial_{1}-\beta \partial_{2}\right\rangle
$$


These distributions are transversal. Let us rectify them, i.e. make a change of coordinates such that $\partial_{1}$ has direction along the first distribution and $\partial_{2}$ along the second. It is possible since the integral curves form a (coordinate) lattice. Now in new coordinate system the structure $j$ has the form

$$
j \partial_{1}=0, \quad j \partial_{2}=0, \quad j \xi=\partial_{1}, \quad j \eta=\partial_{2} .
$$

Here $\xi=\hat{\alpha} \partial_{3}+\hat{\beta} \partial_{4}, \eta=\hat{\gamma} \partial_{3}+\hat{\delta} \partial_{4}$ with some new matrix of functions

$$
\hat{A}=\left(\begin{array}{ll}
\hat{\alpha} & \hat{\beta} \\
\hat{\gamma} & \hat{\delta}
\end{array}\right),
$$

which is nondegenerate in some neighborhood of zero, $\operatorname{det}(\hat{A}) \neq 0$. Now since by the construction the distributions $\left\langle\partial_{1}, \partial_{2}, \xi\right\rangle,\left\langle\partial_{1}, \partial_{2}, \eta\right\rangle$ are integrable we obtain that after some rescaling of rows the matrix $\hat{A}$ depends only on $\left(x_{3}, x_{4}\right)$. In other words $\xi=\lambda_{1}\left(x_{1}, x_{2}\right) \xi_{0}$, $\eta=\lambda_{2}\left(x_{1}, x_{2}\right) \eta_{0}$, where $\xi_{0}, \eta_{0}$ are vector fields projectable to the plane $\mathbb{R}^{2}\left(x_{3}, x_{4}\right)$. Now we have two transversal 1-distributions $\left\langle\xi_{0}\right\rangle$ and $\left\langle\eta_{0}\right\rangle$ on the plane $\mathbb{R}^{2}\left(x_{3}, x_{4}\right)$. Again as above we can change $\xi_{0}$ and $\eta_{0}$ by some factors so that they preserve their direction but begin commute: $\left[\xi_{0}, \eta_{0}\right]=0$. Thus we can make a change of coordinates such that $\xi_{0}, \eta_{0}$ become $\partial_{3}, \partial_{4}$. In this new coordinate system the structure $j$ is given by the formula

$$
j \partial_{1}=0, \quad j \partial_{2}=0, \quad j \partial_{3}=a \partial_{1}, \quad j \partial_{4}=b \partial_{2} .
$$

Now the condition $N_{j}\left(\partial_{3}, \partial_{4}\right)=0$ gives

$$
\left[j \partial_{3}, j \partial_{4}\right]=\frac{\partial b}{\partial x_{1}} \partial_{2}-\frac{\partial a}{\partial x_{2}} \partial_{1}=0 .
$$

Thus $a=a\left(x_{1}, x_{3}, x_{4}\right), b=b\left(x_{2}, x_{3}, x_{4}\right)$ and the lemma is proved.

Remark 3 . The formula of the lemma shows that there are nilpotent structures $j$ with $N_{j}=0$ but which are non-integrable ( $a \neq$ const or $b \neq$ const). However one can check that the condition $(\star), N_{j}=0$, implies the equivalence of the Monge-Ampère equation $\theta=i_{j} \omega=0$ to the third equation of Theorem 1. Actually in symplectic coordinates such that $\operatorname{Ker}(j)=\operatorname{Im}(j)=\left\langle\partial_{p_{1}}, \partial_{q_{2}}\right\rangle$ we have

$$
j \partial_{p_{1}}=0, \quad j \partial_{p_{2}}=a \partial_{p_{1}}+b \partial_{q_{2}}, \quad j \partial_{q_{1}}=c \partial_{p_{1}}+d \partial_{q_{2}}, \quad j \partial_{q_{2}}=0 .
$$

The condition $N_{j}=0$ implies also $\left[a \partial_{p_{1}}+b \partial_{q_{2}}, c \partial_{p_{1}}+d \partial_{q_{2}}\right]=0$ and the identity $\omega(j \xi, \eta)=$ $\omega(\xi, j \eta)$ yields $a=d$. We can suppose the last function is nonzero. Thus $\theta=a d p_{2} \wedge d q_{1}$ and the Monge-Ampère equation can be written in the form

$$
\frac{\partial^{2} u}{\partial q_{1}^{2}}=0
$$

\section{References}

[AG] V. I. Arnol'd, A. B. Givental', Symplectic geometry, in: Dynamical Systems IV, Encyclopaedia Math. Sci. 4, Springer, Berlin, 1990, 1-136.

[Au] T. Aubin, Nonlinear Analysis on Manifolds. Monge-Ampère Equations, Grundlehren Math. Wiss. 252, Springer, New York, 1982. 
[FN] A. Frölicher, A. Nijenhuis, Theory of vector-valued differential forms I, Nederl. Akad. Wetensch. Proc. Ser. A 59 = Indag. Math. 18 (1956), 338-359.

[G] E. Goursat, Leçons sur l'intégration des équations aux dérivées partielles du second ordre à deux variables independantes, tome I, Hermann, Paris, 1896.

[Ha] J. Haantjes, On $X_{m}$-forming sets of eigenvectors, Nederl. Akad. Wetensch. Proc. Ser. A 58 = Indag. Math. 17 (1955), 158-162.

[KLV] I. Krasil'shchik, V. Lychagin, A. Vinogradov, Geometry of jet spaces and nonlinear partial differential equations, Adv. Stud. Contemp. Math. 1, Gordon and Breach, New York, 1986.

[Kr1] B. S. Kruglikov, Some classificational problems in four dimensional geometry: distributions, almost complex structures and Monge-Ampère equations (in Russian), Mat. Sb. 189 no. 11 (1998), 61-74; e-print: http:/xxx.lanl.gov/abs/dg-ga/9611005.

[Kr2] B. S. Kruglikov, Symplectic and contact Lie algebras with an application to MongeAmpère equations (in Russian), Trudy Mat. Inst. Steklov 221 (1998), 232-246; e-print: http:/xxx.lanl.gov/abs/dg-ga/9709004.

[Ku] A. Kushner, Symplectic geometry of mixed type equations, in: The Interplay between Differential Geometry and Differential Equations, V. V. Lychagin (ed.), Amer. Math. Soc. Transl. ser. 2, 167, Amer. Math. Soc., Providence, 1995, 131-142.

[Lie] S. Lie, Gesammelte Abhandlungen, Band VI, Aschehoug, Oslo, and Teubner, Leipzig, 1922.

[Ly1] V. V. Lychagin, Contact geometry and nonlinear second order differential equations, Uspekhi Mat. Nauk 34 no. 1 (1979), 137-165 (in Russian); English transl.: Russian Math. Surveys 34 (1979), 149-180.

[Ly2] V. Lychagin, Lectures on Geometry of Differential Equations, Ciclo di conferenze tenute presso il Dipartimento di Metodi e Modelli Matematici per le Scienze Applicate Università "La Sapienza", Roma, 1992.

[LRC] V. V. Lychagin, V. N. Rubtsov, I. V. Chekalov, A classification of Monge-Ampère equations, Ann. Sci. École Norm. Sup. (4) 26 (1993), 281-308.

[M] T. Morimoto, Le problème d'équivalence des équations de Monge-Ampère, C. R. Acad. Sci. Paris Sér. A-B 289 (1979), A25-A28.

[NN] A. Newlander, L. Nirenberg, Complex analytic coordinates in almost-complex manifolds, Ann. Math. (2) 65 (1957), 391-404.

[R] A. Rakhimov, Singularities of Riemann invariants, Funktsional. Anal. i Prilozhen. 27 no. 1 (1993), 46-59 (in Russian); English transl.: Funct. Anal. Appl. 27 (1993), 39-50.

[S] S. Sternberg, Lectures on Differential Geometry, Prentice-Hall, Englewood Cliffs, 1964.

[T] N. Tanaka, On differential systems, graded Lie algebras and pseudogroups, J. Math. Kyoto Univ. 10 (1970), 1-82. 